\title{
Experimental Study on Directional Fracture Blasting of Cutting Seam Cartridge
}

\author{
Renshu Yang ${ }^{1,2,3}$ and Jinjing Zuo $\mathbb{D}^{1}$ \\ ${ }^{1}$ School of Mechanics and Civil Engineering, China University of Mining and Technology (Beijing), Beijing 100083, China \\ ${ }^{2}$ State Key Laboratory for Geomechanics and Deep Underground Engineering (Beijing), Beijing 100083, China \\ ${ }^{3}$ School of Civil and Environmental Engineering, University of Science and Technology Beijing, Beijing 100083, China
}

Correspondence should be addressed to Jinjing Zuo; cumtbzjj@163.com

Received 12 December 2018; Revised 16 April 2019; Accepted 6 May 2019; Published 23 May 2019

Academic Editor: Itzhak Green

Copyright (C) 2019 Renshu Yang and Jinjing Zuo. This is an open access article distributed under the Creative Commons Attribution License, which permits unrestricted use, distribution, and reproduction in any medium, provided the original work is properly cited.

\begin{abstract}
Since the cutting seam cartridge has sound directional breaking effect, in order to explore its blasting wave mechanism, an experimental study was conducted on the blast wave of a cutting seam cartridge using a high-speed laser schlieren system and air overpressure test system. The research results show that during cartridge explosion, the cutting seam pipe can effectively control the energy release and detonation gas dynamic behaviors. Additionally, the cartridge blast wave and the detonation gas remain highly symmetrical in expansion form. The blast wave first propagates along the cutting seam direction, and the detonation gas initially expands from the seam in the direction of the cutting seam. The pressure in the cutting seam direction is higher than that in the noncutting seam direction. The blast wave change of the whole flow field of the cutting seam cartridge was numerically simulated and was basically consistent with the results of the schlieren test in distribution form. The presence of a cutting seam prolongs the blast wave effect. During expansion of the cutting seam pipe under the influence of a blast wave, the inner wall of the cutting seam pipe is mainly subject to tensile stress. When the tensile force applied to the inner wall reaches the local yield limit, the cutting seam pipe fails.
\end{abstract}

\section{Introduction}

Blasting technology with directional control for a cutting seam cartridge is widely and effectively applied in engineering fields like deep rock projects, tunnel excavation, slope excavation, and precious stone mining [1-3]. A cutting seam cartridge is the placement of an explosive in a tube shell with a certain strength and with kerf seams on both sides of the shell, as shown in Figure 1. The cutting seam cartridge is used to control the propagation direction of explosion shock wave and detonation gas, to achieve directionally controlled detonation.

Fourney et al. [4,5] used a pipe with cutting seam in the blast hole to control the directional cutting seam direction after blasting and experimentally demonstrated that cracks could be obtained in the designated direction with this charging method, thus controlling the fracture. Yang et al. [6-9] studied the dynamic process of use of a cutting seam cartridge driving crack expansion with dynamic caustics and performed SEM scanning of the fracture surface to study the damage mechanism. Wang [10] simulated rock damage by the cutting seam cartridge with different coupling coefficients with LS-DYNA. Wang et al. [11-13] used a highspeed schlieren system and numerical study to examine the electrical explosion of aluminum wire in a vacuum or in the air. Forde et al. [14] studied the impact performance of borosilicate glass (heat-resistant glass) under a light air gun with the schlieren system. Kellenberger and Ciccarelli $[15,16]$ studied the blast wave transmission process on a barrier with a high-speed schlieren system. Li et al. $[17,18]$ studied the deflagration-to-detonation transition (DDT) of the incident wave in a bent pipeline using a schlieren system. Damazo et al. [19] and Mazaheri et al. [20-22] studied the transmission behavior of the impact wave during air explosion and the reflection law on the wall. Gerasimov et al. [23] studied shock wave propagation of an explosive 


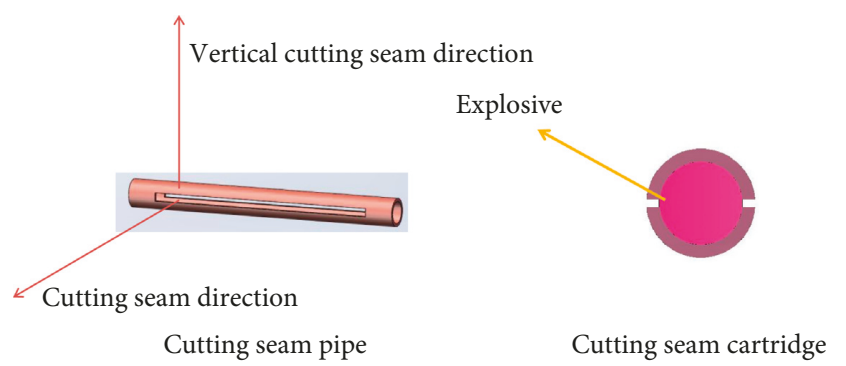

FIgURE 1: Cutting seam cartridge.

material with plastic filler, using measurements of the air shock wave propagation to estimate a maximum pressure in different sections of the compression zone. Larcher et al. [24] studied the explosion-proof grade of laminated glass windows and facades by numerical simulation and provided an initial attempt at possible standardization of these kinds of numerical simulations.

However, most studies of blast wave behavior of a cutting seam cartridge have mostly relied on extrapolation from explosive detonation theory and there has been no systematic experimental study of the blast wave behavior of a cutting seam cartridge. In this study, experiment and numerical analysis were combined to describe the dynamic evolution mechanism of the blast wave of a cutting seam cartridge.

\section{Experimental Details}

2.1. Test System. A high-speed laser schlieren system was used to capture the structural features of the seam cartridge during the front propagation of the detonation wave, and an air overpressure test system was used to capture the pressure change in the cutting seam direction and the vertical direction and analyze the difference of pressure in these two directions.

Figure 2 shows the comprehensive test system adopted in the experiment. The experimental system consists of a laser device, beam expander, concave radioscope, cutter, and high-speed camera. The monochrome laser from the laser device forms a point source laser after an optical lens, and the point source laser is the light source of beam divergence after adjustment of focus size by the hole. The wavelength of the laser used is $650 \mathrm{~nm}$, and the concave radioscope diameter is $300 \mathrm{~mm}$. This light source is sent to the concave reflector after the mirror plane and becomes parallel light after the concave mirror. The light carries the flow field information after the test section and then is focused on the cutter by the receiving concave reflector. Part of this light is removed by the cutter, and then the camera positioned after the filter can be used to obtain the schlieren photographs.

The air overpressure test system consists of the pressure sensor, charge amplifier, and oscilloscope. Pressure sensors are arranged at equal distance in the cutting seam direction and the vertical direction. The formula to measure the blast wave peak overpressure of the cutting seam cartridge is

$$
P_{\mathrm{m}}=\frac{V_{\max }}{K \cdot S_{q}}
$$

where $V_{\max }$ is the peak voltage indicated on the oscilloscope, $\mathrm{mV}$; $K$ is the sensitivity of the charge amplifier, $\mathrm{mV} / \mathrm{pC}$, and is set to $10 \mathrm{mV} / \mathrm{pC}$ in the experiment; and $S_{q}$ is the charge sensitivity of the sensor, $\mathrm{pC} / \mathrm{MPa}$. The charge sensitivity is first calibrated and the pressure charge sensitivity of the sensor is $S_{q}=297.5 \mathrm{pC} / \mathrm{MPa}$.

2.2. Description of Experiment. Figure 3 shows the schlieren pattern of cutting seam cartridge. The experimental cartridge is designed as follows: the cutting seam material is stainless steel, outer diameter is $12 \mathrm{~mm}$, inner diameter is $6 \mathrm{~mm}$, wall thickness is $3 \mathrm{~mm}$, and the cutting seam width is $2 \mathrm{~mm}$. The selected explosive is primary explosive (DDNP), which is highly sensitive to spark, and $200 \mathrm{mg}$ was used as the charge amount. During detonation, the explosive is detonated by the high voltage discharge of a twisted enameled wire and the detonation point is at the cartridge center. A Fastcam SA5 highspeed camera is set at the end of the cutter, and the shooting speed of the camera is 100000 pictures/second. The image resolution is $320 \times 190$, and the number of pixels is 60800 .

\section{Experimental Results of Blast Wave of the Cutting Seam Cartridge}

3.1. Blast Wave Process of the Cutting Seam Cartridge. Figure 4 shows the detonation wave process of the cutting seam cartridge. At $20 \mu$ s, the blast shock wave at two cutting seams expanded rapidly along the two cutting seam directions, with the blast waves distributed in the "spindle" shape at the two seams. Due to the small diameter of the cartridge, the detonation waves flow rapidly around a body to each other's region from the two seam directions. At $60 \mu \mathrm{s}$, the detonation gas started to separate from the blast shock wave, and the detonation gas and the shock wave still propagate synchronously in that direction. At $120 \mu \mathrm{s}$, the blast shock wave in the cutting seam direction reached the pressure sensor measuring point, but the shock wave in the vertical cutting seam direction had not yet reached the measuring point. The detonation gas expanded to the farthest point in the vertical cutting seam direction, and the detonation gas expansion gradually slowed. At $160 \mu \mathrm{s}$, the distance between the blast shock wave and detonation gas had gradually widened, and the blast shock wave in the vertical cutting seam direction reached the sensor position. At $330 \mu$ s, the blast wave had expanded past the field of view, and the detonation gas changed from slow to rapid expansion in the cutting seam direction. However, in the vertical direction, the position did not change obviously. After $560 \mu \mathrm{s}$, the detonation gas mainly expanded in an I-shaped form along the cutting seam direction in the form of twin status.

3.2. Blast Wave Front Speed of the Cutting Seam Cartridge. Figure 5 shows the curve of the changes of the blast wave and detonation gas expansion speed and displacement with time 


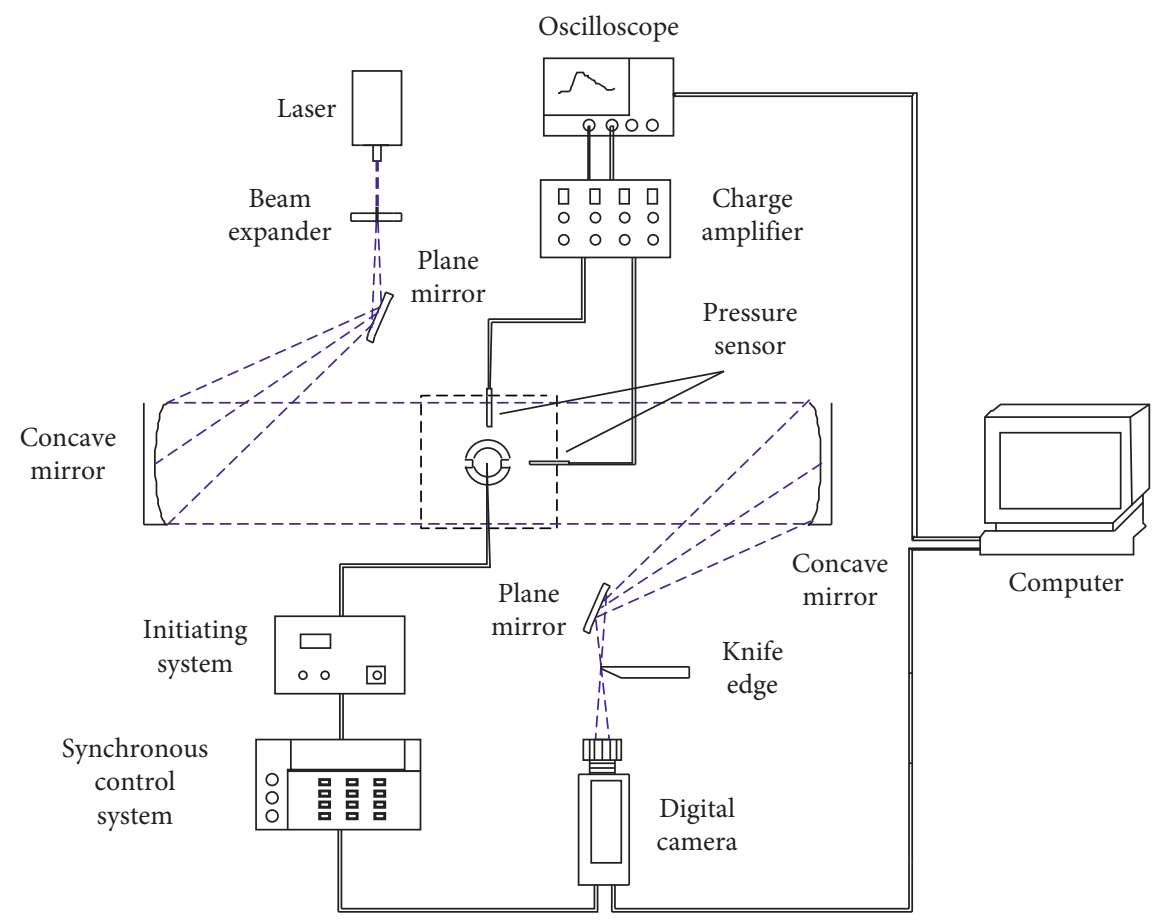

FIGURE 2: High-speed schlieren photography experimental system.

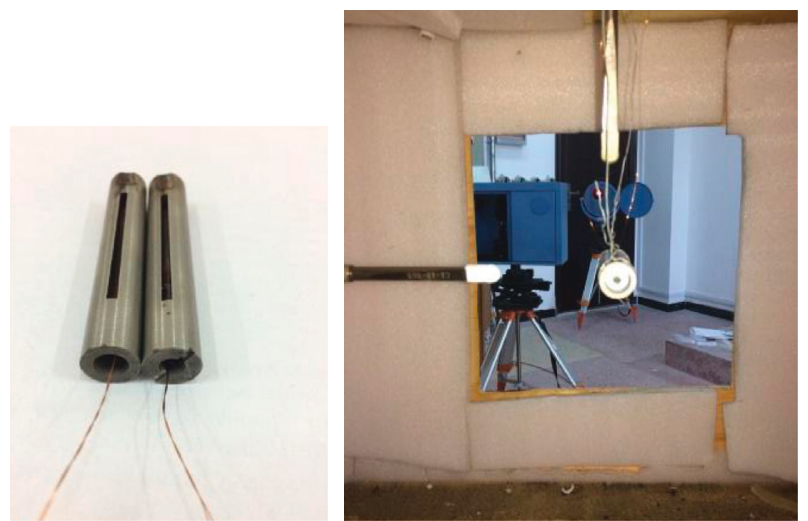

Figure 3: Cutting seam cartridge used in tests with high-speed schlieren photography.

( $S$ indicates speed, $D$ indicates displacement, kerf indicates cutting seam direction, and vertical-kerf indicates the direction vertical or perpendicular to the cutting seam direction). As suggested in Figure 5(a), the speed of the blast wave in the cutting seam direction is faster in the cutting seam direction than that in the vertical cutting seam. The blast wave in the vertical cutting seam direction is caused by the blast wave streaming in the cutting seam direction, so the initial speed is $0 \mathrm{~m} / \mathrm{s}$. The blast wave in the two directions decreases with the same trend, so there is a rapid speed decrease in the near region, and the speed decrease is less in the middle region and still slower in the far regions.

Figure 5(b) shows the curve of the changes of detonation gas displacement speed with time. In the first $60 \mu \mathrm{s}$, the detonation gas has relatively vague status and it is impossible to determine its expansion displacement; the curve starts at $60 \mu \mathrm{s}$. From
60 140 $\mu$ s, the propagation speed of gases in these two direction decreases rapidly and the speed in the cutting seam direction is larger than that in the vertical cutting seam direction. From $140 \sim 250 \mu \mathrm{s}$, the detonation gas expansion speeds in the two directions are clearly different. Stagnation occurs in the cutting seam direction, and then it suddenly increases to about $200 \mathrm{~m} / \mathrm{s}$. In the vertical cutting seam direction, the detonation gas expansion speed decreases to 0 and then decreases rapidly to $-130 \mathrm{~m} / \mathrm{s}$ (here the symbol "-" means opposite to the original direction). This change in direction is due to the "retraction" of detonation gas in the vertical cutting seam direction.

As shown from the above experimental results, the blast wave of cutting seam cartridge preferentially propagates along the cutting seam direction and then the detonation gas is released in the I-shaped form along the cutting seam direction. Figure 6 shows the physical model of the blast wave of the cutting seam cartridge. After detonation of the cartridge, the initial blast wave occurs in the cutting seam direction, which then propagates along the cutting seam direction, then diffracts, and propagates along the outer wall of the cutting seam pipe.

Figure 7 shows the physical model of the detonation gas of the cutting seam cartridge. The detonation gas spreads from the cutting seam. After some time of propagation, the shock wave front becomes completely separated from the detonation gas, which expands to the limit volume. The detonation gas displacement in the vertical cutting seam direction reaches maximum value. The detonation gas then exhibits retraction in the vertical direction, resulting in compression of the detonation gas so that it expands along the cutting seam direction. During this process, the detonation gas exhibits almost stagnant status along the cutting seam direction and then expands along the cutting seam direction. The detonation gas is released in an I-shaped form along the cutting seam direction. 


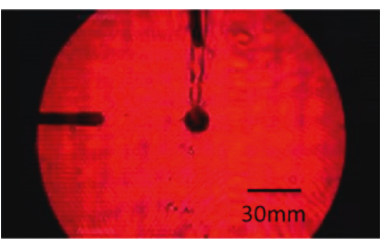

(a)

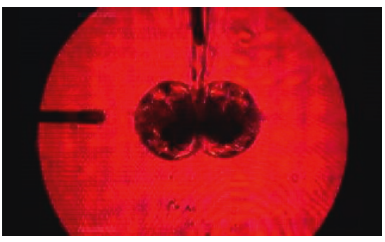

(c)

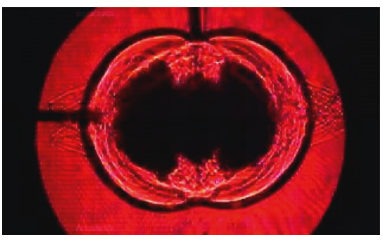

(e)

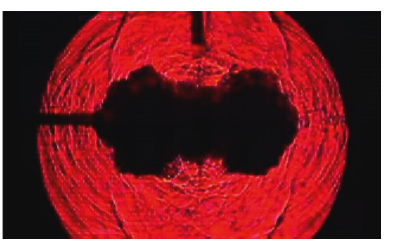

(g)

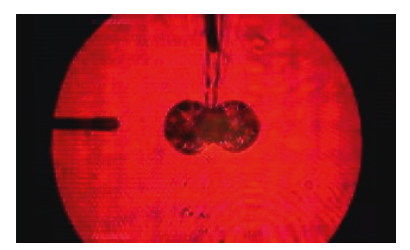

(b)

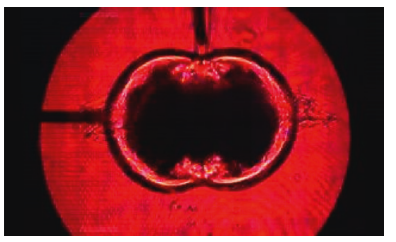

(d)

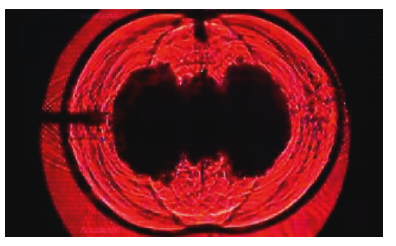

(f)

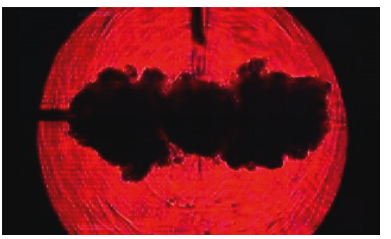

(h)

Figure 4: Process picture of slotted cartridge blast wave. (a) $0 \mu \mathrm{s}$. (b) $20 \mu \mathrm{s}$. (c) $60 \mu \mathrm{s}$. (d) $120 \mu \mathrm{s}$. (e) $160 \mu \mathrm{s}$. (f) $220 \mu \mathrm{s}$. (g) $330 \mu \mathrm{s}$. (h) $560 \mu \mathrm{s}$.

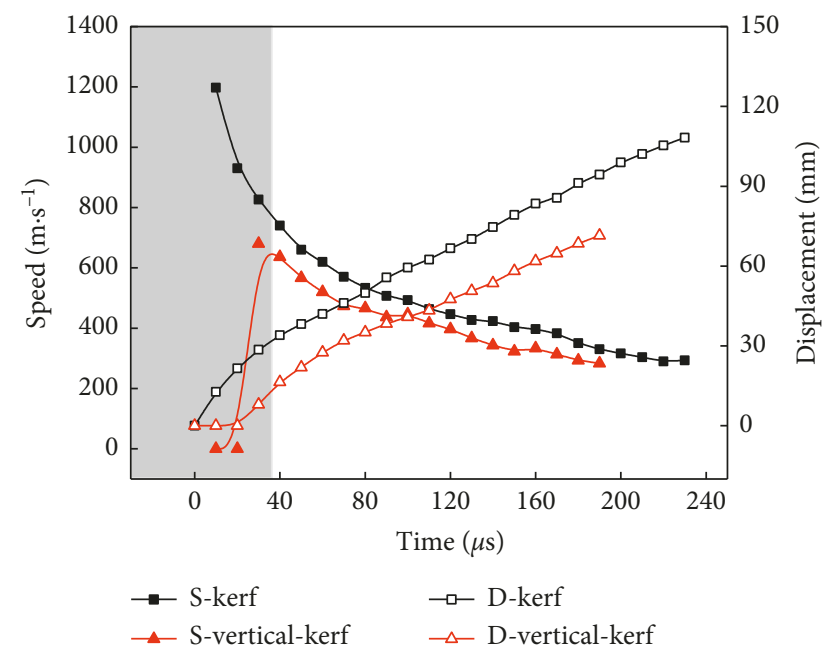

(a)

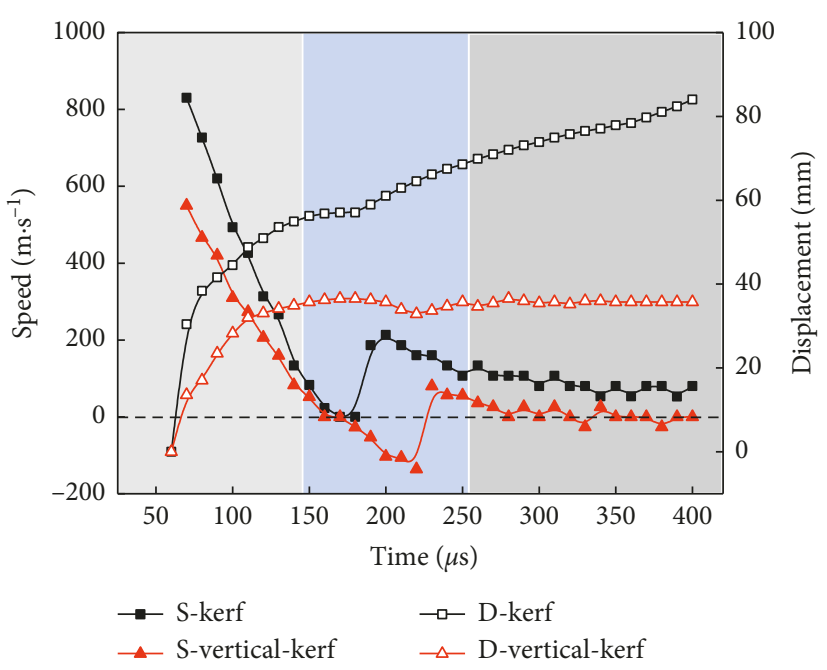

(b)

FIGURE 5: Curve of cutting seam cartridge explosion shock wave and explosion gas displacement-speed over time. (a) Explosive shock wave. (b) Detonation gas.

The analysis above shows the movement speed of the wave front and detonation gas. However, this speed reflects the weakening of the wave, and the speed determines the shattering capability, or brisance, which reflects the change of impulse. In this way, the advantageous detonation gas accumulates in the cutting seam direction and impacts the medium. The high strength stress concentration of local effect directly leads to directional medium damage. 


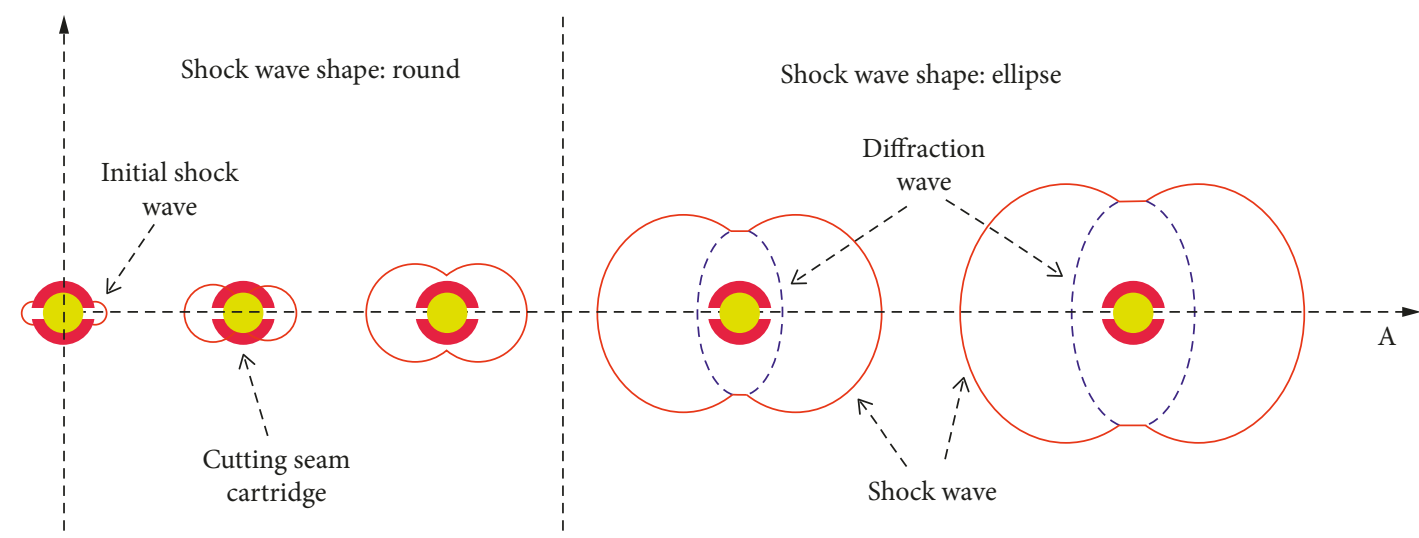

FIgURe 6: Physical model of explosive wave in cutting seam cartridge.

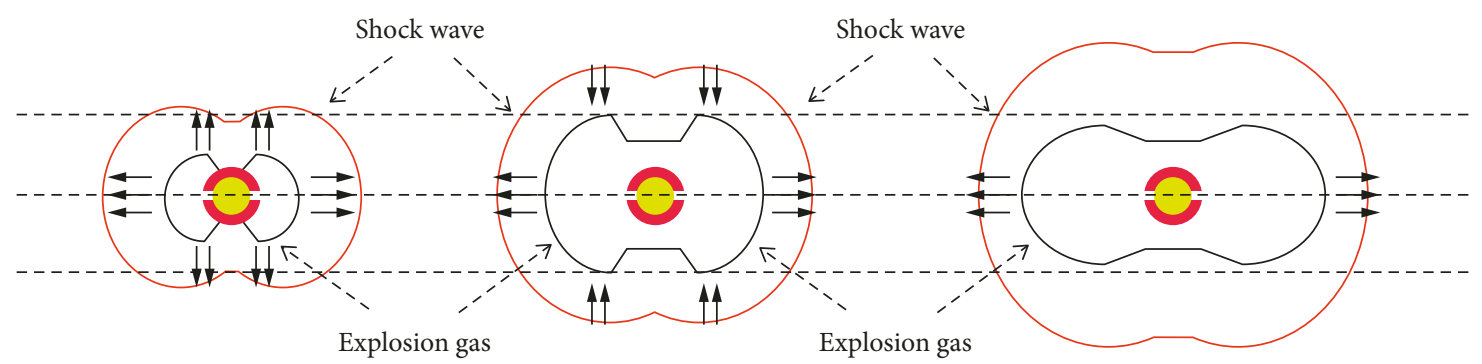

Figure 7: Physical model of explosive gas in cutting seam cartridge.

\section{Experimental Results of Cutting Seam Cartridge Overpressure Test}

4.1. Cutting Seam Cartridge Blast Wave Shape with Two-Point Synchronous Test. Figure 8 shows the curve of air blast wave pressure of the cutting seam cartridge at different measuring points. Figure 8(a) shows the time-distance curve of air blast wave measured at $5 \mathrm{~cm}$ from the cartridge center. As shown in the figure, the pressure signal is first captured in the cutting seam direction. The vertical cutting seam direction lags $40 \mu \mathrm{s}$ for the same distance measuring point, which is consistent with the schlieren observation results that the propagation of air blast wave in the cutting seam direction is faster than that in vertical cutting seam direction. The pressure peak values in the cutting seam direction and vertical cutting seam direction are $1.34 \mathrm{MPa}$ and $0.52 \mathrm{MPa}$, respectively. The blast wave overpressure in the cutting seam direction is 2.57 times that in vertical cutting seam direction. Figure 8 (b) shows the air blast wave overpressure time-distance curve measured $10 \mathrm{~cm}$ from the cartridge center. The time interval of the pressure curve in two directions is 90; the pressure peak values in the cutting seam direction and vertical cutting seam direction are $0.52 \mathrm{MPa}$ and $0.24 \mathrm{MPa}$, respectively. The blast wave overpressure in the cutting seam direction is 2.17 times that in the vertical cutting seam direction. The overpressure in the vertical cutting seam direction occurs twice, and the overpressure peak value is $0.034 \mathrm{MPa}$.

4.2. Cutting Seam Cartridge Overpressure Attenuation Law. The main factors affecting air overpressure are explosive amount and measuring distance. A single variable experiment was conducted for these two factors, and the overpressure values of the cutting seam cartridge were measured. Using the least square method, we obtained the curve of air blast wave pressure attenuation of the cutting seam cartridge in two directions and the fitting curve is shown in Figure 9.

Figure 10 shows the fitting curve of the cutting seam cartridge peak pressure ratio between the cutting seam direction $\left(P_{\text {kerf }}\right)$ and the vertical cutting seam direction $\left(P_{\text {ver-kerf }}\right)$. As shown in the curve, the pressure ratio of these two pressures in the region close to the cutting seam cartridge decreased with increasing proportion distance, and the ratio decrease amplitude declined with increasing pressure ratio. This suggests that the overpressure in the cutting seam direction is obviously different from that in the vertical cutting seam direction closest to the cartridge, and this difference becomes more apparent closer to the cartridge.

4.3. Measurement Errors Analysis. In the schlieren experiment, there may be error due to the simplification of calculations, the loading method, the specimen processing, and in parameter measurement.

Explosive loading was used as the experimental loading method. Due to the instantaneity of explosive load and the relationship between the charge and cutting seam pipe, the position of the detonation point will result in errors in the test results. Therefore, a strict operation process and accurate control charge are required to reduce error, with careful selection of the detonation point position.

One of the key problems in the test technology is controlling the optimal width and length of the cutting 


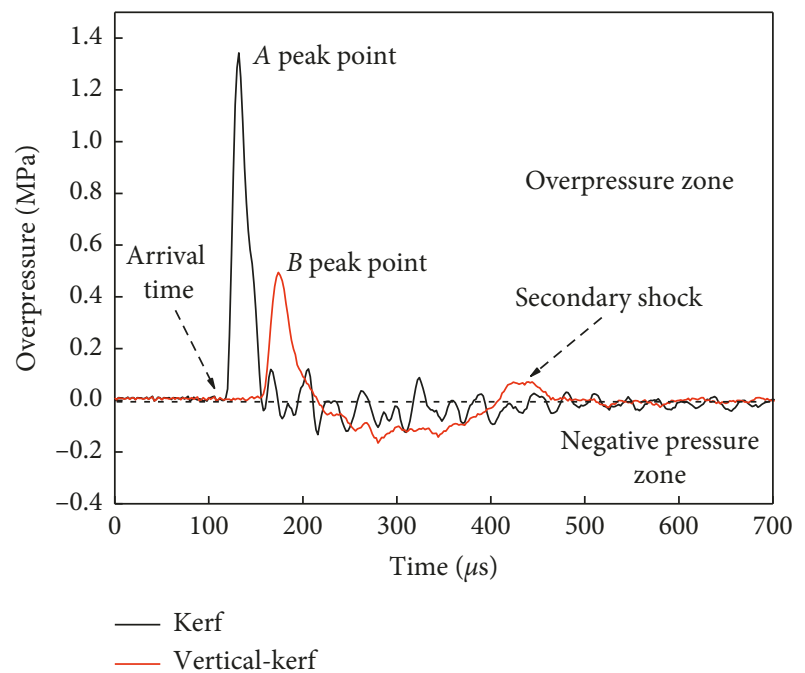

(a)

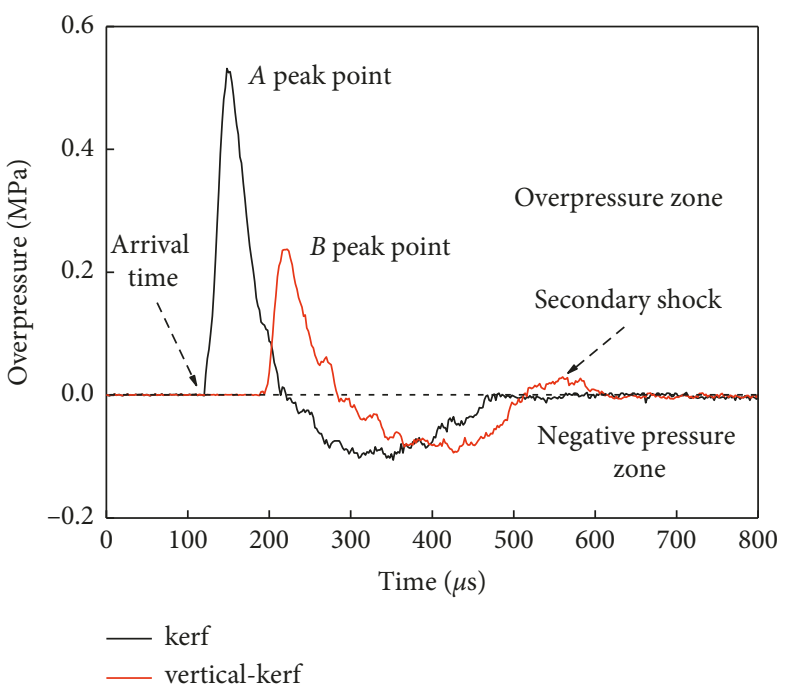

(b)

FIGURE 8: Curve of explosion shock wave overpressure of cutting seam cartridge. (a) $5 \mathrm{~cm}$ from the cartridge center. (b) $10 \mathrm{~cm}$ from the cartridge center.

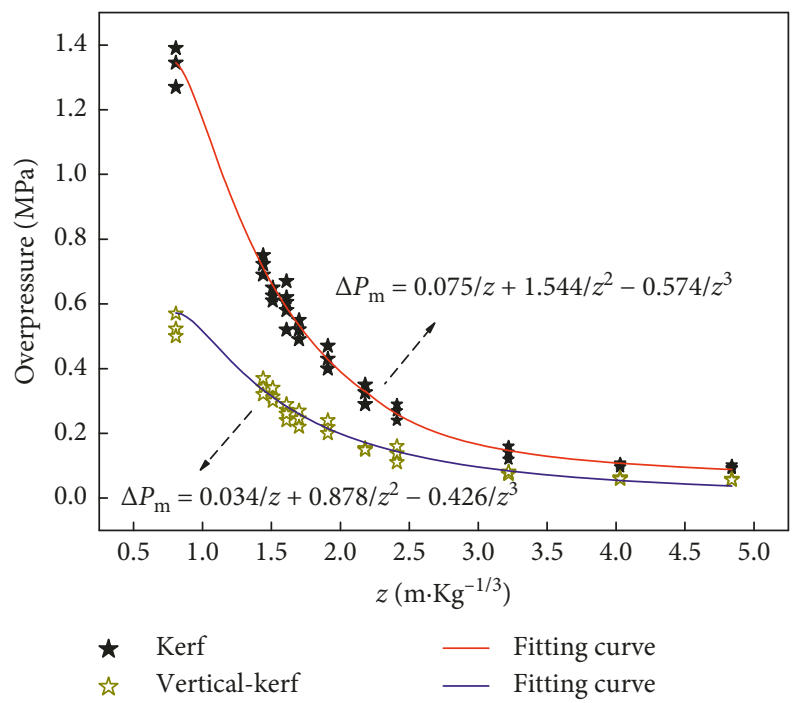

Figure 9: Pressure attenuation curve of the cutting direction and the vertical direction of the cutting seam cartridge.

seam pipe. If the control is not good, there will be a deviation between the blast wave and the detonation gas, producing errors in pressure measurement. In this experimental setup, a mechanical wire cutting method was used and controlled by a computer program to ensure accuracy.

According to the calculation formula of the overpressure of the air shock wave, the necessary parameters to be measured are $V_{\max }$ and $S_{q}$. The main error in the measurement comes from the measurement of $V_{\max }$ on the oscilloscope, so this measurement must be particularly careful. When calculating the expansion speed of air shock wave and detonation gas, manual measurement is particularly error-prone, so multiple measurements should be made and the average value obtained.

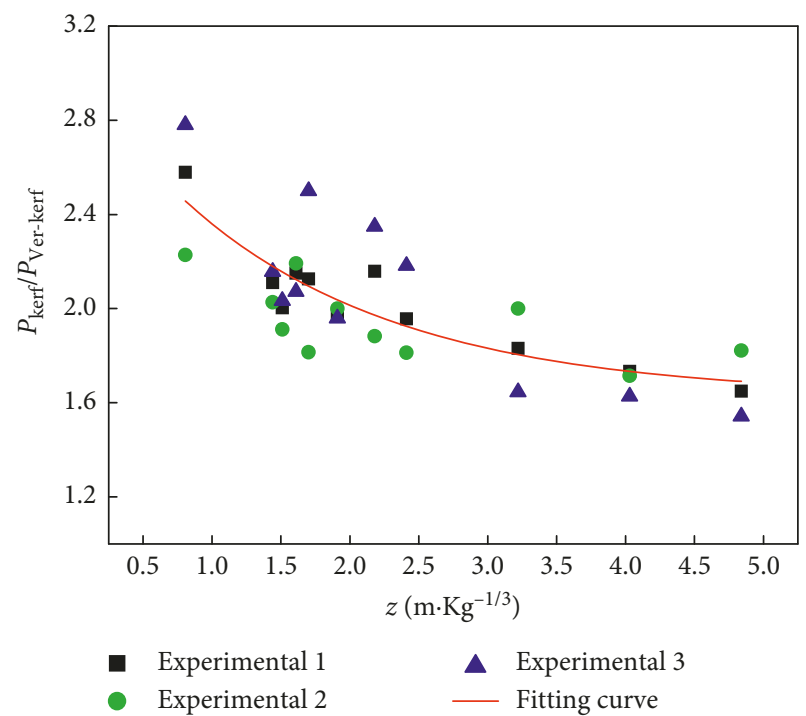

FIGURE 10: Curve of the peak pressure ratio between cutting seam direction and vertical cutting seam direction.

According to the principle of system test error, the pressure conversion relation is

$$
P_{\mathrm{m}}=V_{\max } \cdot K^{-1} \cdot S_{q}^{-1} \text {. }
$$

According to the principle of relative error, the relative error of pressure measurement is

$$
\left|\frac{\Delta P}{P}\right|=\left|\frac{\Delta V_{\max }}{V_{\max }}\right|+\left|\frac{\Delta K}{K}\right|+\left|\frac{\Delta S_{q}}{S_{q}}\right| .
$$

According to the accuracy of each part of the test system, $\Delta K / K=1.5 \%, \Delta S_{q} / S_{q}=1.5 \%$, and storage oscilloscope sensitivity is $0.52 \%$. Therefore, the systematic error is $\Delta P / P=3.52 \%$. 


\section{Numerical Simulation of Cutting Seam Cartridge Blast Wave}

Because the schlieren experiment could not observe the initial state of the blast wave at the cutting seam, to more deeply explore the whole field domain evolution mechanism of the cutting seam cartridge blast wave, a numerical calculation model was established for the cutting seam cartridge to allow numerical simulation of cartridge blast wave.

The numerical analysis software adopts Autodyn, using finite element analysis to solve highly nonlinear dynamics problems of solids, fluids, gases, and their interactions. This approach can simulate all kinds of impact response and explosion problems.

\subsection{Finite Element Model and Material Parameters. To study} the explosion process of a cutting seam pipe under the "explosive-cutting seam pipe-air" effect, the explosion model of cutting seam cartridge is presented in Figure 11. One hundred twenty-six thousand units are included in total. The explosive diameter is $26 \mathrm{~mm}$, the cutting seam pipe thickness is $2 \mathrm{~mm}$, and the cut is $4 \mathrm{~mm}$. Since the cutting seam pipe is a thin-wall shell structure, the cutting seam pipe wall experiences significant deformation and even damage under a strong dynamic explosion load. During numerical calculation, the ALE algorithm is used to describe the cutting seam pipe to prevent excessive mesh distortion causing the calculation to stop. The Euler algorithm is adopted for explosive and air. Fluid structure interaction method (FSI) is adopted between the cutting seam pipe and air [25] to simulate the explosive blast product and the interaction between the air blast wave and the cutting seam pipe and the simulated blast hole. The air boundary condition is a nonreflecting boundary. The cutting seam pipe is steel. The shock status equation and Steinberg-Guinan constitutive model are employed, and the main strain failure and erosion are set.

Air is modeled as an ideal gas. Therefore, the equation of state of the ideal gas is defined by the gamma law relation as formula (4):

$$
P=(\gamma-1) \frac{\rho}{\rho_{0}} E
$$

where $P$ is the pressure and $\gamma$ is the adiabatic index, $\gamma=1.4$. The air density was $1.225 \mathrm{~g} \cdot \mathrm{m}^{-3}$; the reference temperature was $288.2 \mathrm{~K}$; and the specific heat was $717.6 \mathrm{~J} \cdot \mathrm{Kg} \cdot \mathrm{K}^{-1}$.

The explosive is PETN with density of $0.88 \mathrm{~g} \cdot \mathrm{cm}^{-3}$, and the equation of state JWL is shown in formula (5):

$$
P=A\left(1-\frac{\omega}{R_{1} V}\right) e^{-R_{1} V}+B\left(1-\frac{\omega}{R_{2} V}\right) e^{-R_{2} V}+\frac{\omega E_{0}}{V},
$$

where $V$ is the relative volume; parameters $B, A, R_{1}, R_{2}$, and $\omega$ are determined constants; $P$ is the pressure; and $E_{0}$ is the initial internal energy density. The JWL state parameters of PETN are shown in Table 1.

5.2. Blast Wave Propagation Process of Cutting Seam Cartridge. Figure 12 shows the simulation of blast wave

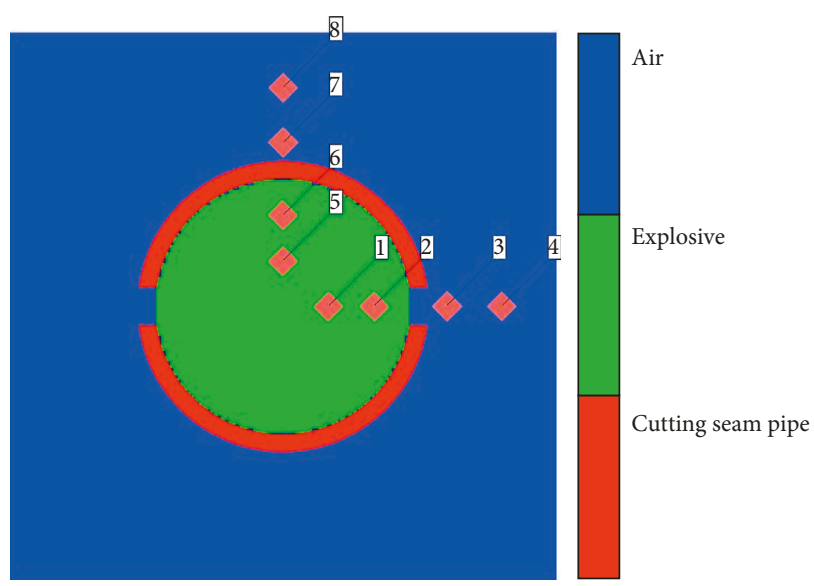

FIGURE 11: Calculation model.

propagation process in the air free field. The blast wave distribution obtained from numerical simulation is very consistent with the high-speed schlieren test results in terms of form. An important difference is that the explosive used here is PETN explosive, which is different from the DDNP used in the schlieren test, so the simulation results differ from the test results in the time scale.

When the initial explosion wave reaches the inner wall of the cutting seam pipe, the blast wave at the cutting seam propagates and instantaneously flies off from the cutting seam. At this time, the blast wave interacts with the whole cutting seam pipe and the pipe wall is instantaneously loaded, producing a high strain rate on the pipe wall, leading to high expansion of the cutting seam pipe. During the whole expansion process, some of the detonation gas and blast wave propagate from the cutting seam, as shown by the speed vector chart. During the subsequent wave process, the blast wave continues to act on the cutting seam pipe, causing the pipe to expand. Meanwhile, the blast wave propagates along the cutting seam direction, with part streaming along the outer wall of the cutting seam pipe and part impacting the surrounding medium. The overall propagation process is consistent with the high-speed schlieren test results. The cutting seam cartridge blast wave in the free field is unconstrained in the near zone and fully reflects the propagation of detonation at the cutting seam. As also suggested in the figure, when the blast wave contacts the inner wall of the cutting seam pipe, it can initially propagate to the air from the cutting seam pipe since the pipe orifice is an air field but reflects on the inner wall of cutting seam pipe at both sides. The reflected blast wave compresses the detonation gas to accelerate expansion from the cutting seam pipe orifice, resulting in the formation of a jet flow effect along the cutting seam direction.

Figure 13(a) shows the pressure change at four measuring points in the cutting seam direction. Points 1 and 2 indicate the internal observation points of the cutting seam cartridge. There are two peaks at $25 \mu \mathrm{s}$; the first is caused by the initial wave and the second is due to the pressure wave generated by the explosion fluctuation that is reflected in the inner wall of the slit pipe back to the center of the explosion source. As shown in the effective stress diagram presented in 
TABle 1: JWL parameters for PETN.

\begin{tabular}{lcccccccc}
\hline$\rho_{0}\left(\mathrm{~g} \cdot \mathrm{cm}^{-3}\right)$ & $D\left(\mathrm{~m} \cdot \mathrm{s}^{-1}\right)$ & $A(\mathrm{GPa})$ & $B(\mathrm{GPa})$ & $R_{1}$ & $R_{2}$ & $\omega$ & $E_{0}(\mathrm{GPa})$ & $P_{\mathrm{C}-\mathrm{J}}(\mathrm{GPa})$ \\
\hline 0.88 & 3900 & 314.5 & 2.78 & 4.2 & 0.8 & 0.26 & 4.89 & 7.6 \\
\hline
\end{tabular}
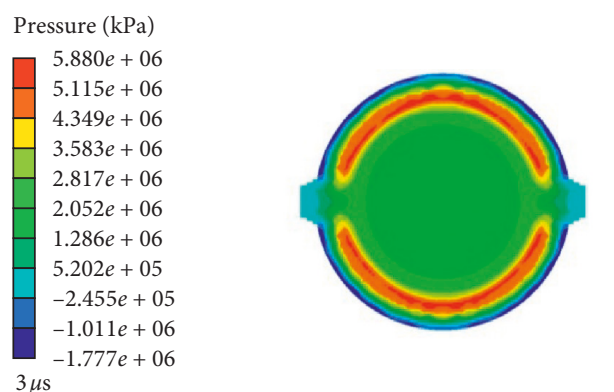

Pressure $(\mathrm{kPa})$

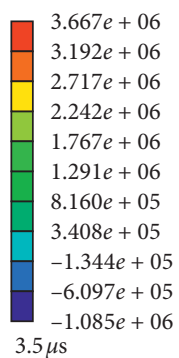

Pressure $(\mathrm{kPa})$

$3.708 e+06$
$3.259 e+06$

$2.810 e+06$

$2.360 e+06$

$1.911 e+06$

$1.462 e+06$

$1.013 e+06$

$5.637 e+05$

$1.145 e+05$

$-3.347 e+05$

$-7.840 e+05$

$4 \mu \mathrm{s}$

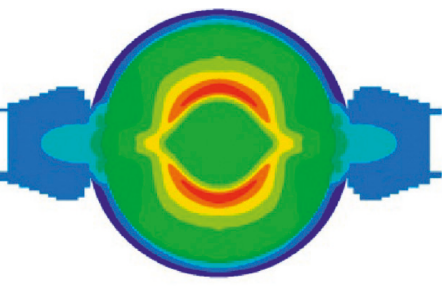

(a)
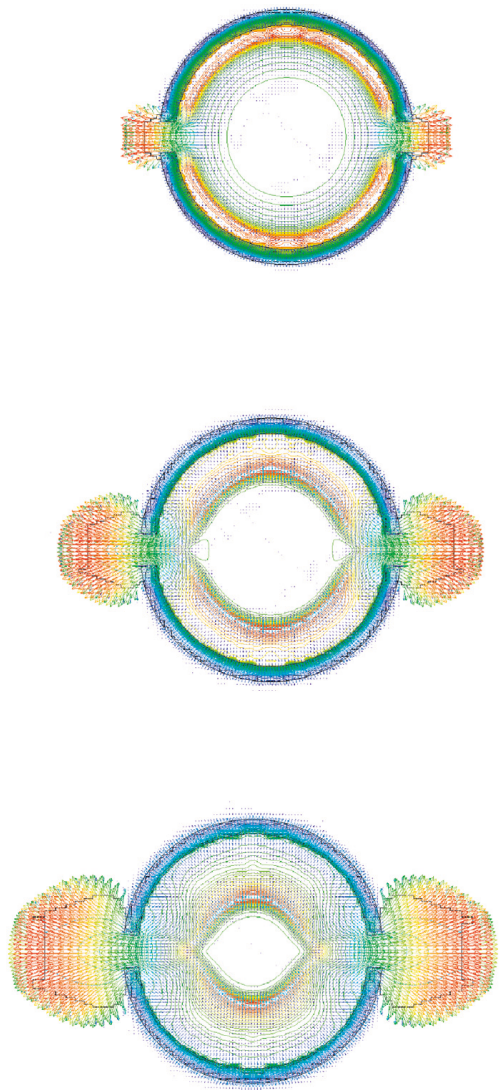

(b)
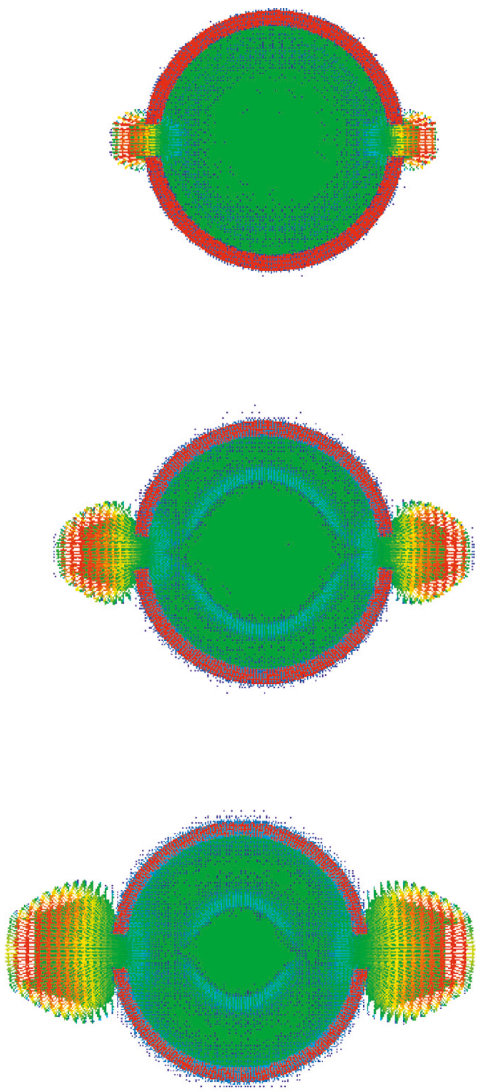

(c)

Figure 12: Blast wave propagation of cutting seam cartridge in free field. (a) Effective stress. (b) Pressure line. (c) Velocity vector.

Figure 12(a), the peak of the secondary pressure wave is the largest closest to the explosion source and is decreased in the far zone. The second fluctuation of measuring point 1 appears later than that of point 2. Points 3 and 4 in Figure 13(a) are the far-field measuring points in the direction of the slit, and the amplitude of the initial wave of the explosion gradually decreases at these points. The variation laws of points 5 and 6 are basically the same as those at the corresponding distances, points 1 and 2 (cutting direction), except that the magnitude of the fluctuation is different. The first peak pressure is the same, and the second peak pressure is greater for points 5 and 6 than the pressure values at measuring points 1 and 2 due to the reflection compression of the slitted tube. Points 7 and 8 show the fluctuation of the far direction of the slit direction, which is very small. The pressure peak for point 3 is 2.86 times that of point 7 and the pressure peak for point 4 is 2.61 times that of point 8 , which is in accordance with the experimental rule obtained in Figure 10.
5.3. Dynamic Response Analysis of the Cutting Seam Pipe. Figure 14 shows the dynamic pressure time-sequence chart of the cutting seam pipe. There is obvious plastic deformation of the cutting seam pipe under dynamic explosive load. During the initial phase of the explosion, pressure is produced on the inner wall of the cutting seam pipe, reaching maximum pressure at the cutting seam pipe. After full explosive reaction, the explosion wave acts on the inner wall of the cutting seam pipe and the pressure on the inner wall exceeds that at the cutting seam. As the detonation gas propagates from the cutting seam pipe to the outside, the pressure wave spreads to the whole cutting seam pipe wall, the cutting seam pipe shell makes expands towards the outside under the pressure of the explosion, the pressure wave is transmitted to the inner part of the cutting seam pipe, and a single rarefaction wave is reflected into the detonation gas. When the blast compression wave within the cutting seam pipe propagates to the free face of the outer wall, it is reflected to a tensile unloading wave, which propagates in the cutting seam pipe shell to the interface between the detonation gas and the 


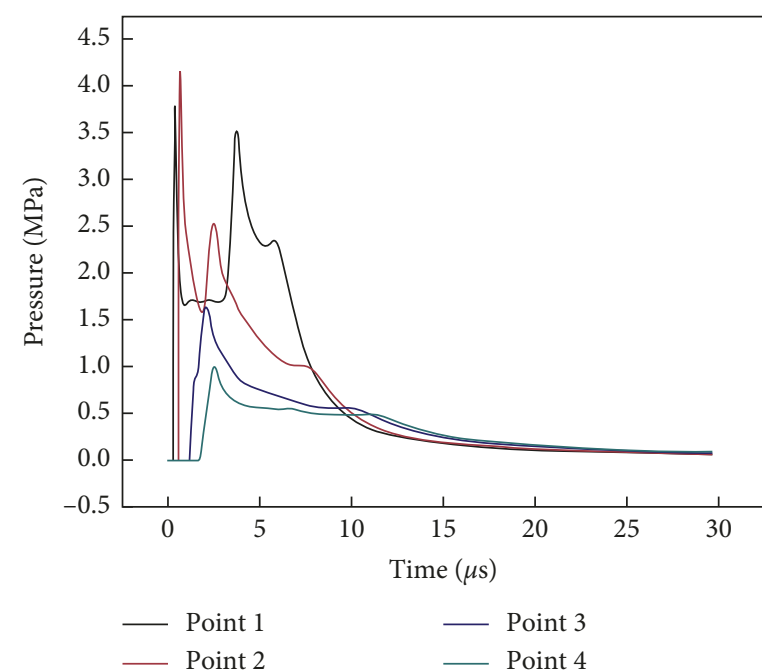

(a)

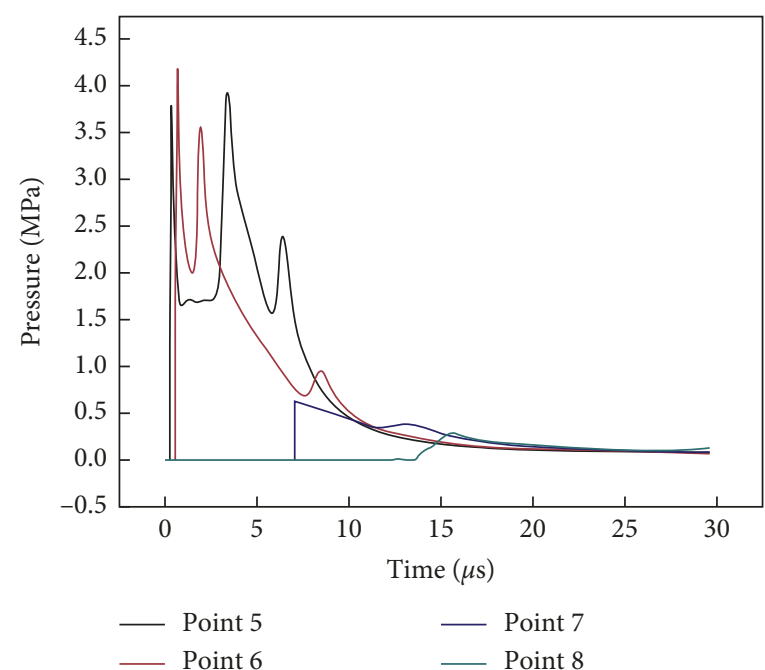

(b)

Figure 13: Blast wave pressure versus time in two directions in free field. (a) Cutting seam direction. (b) Vertical cutting seam direction.

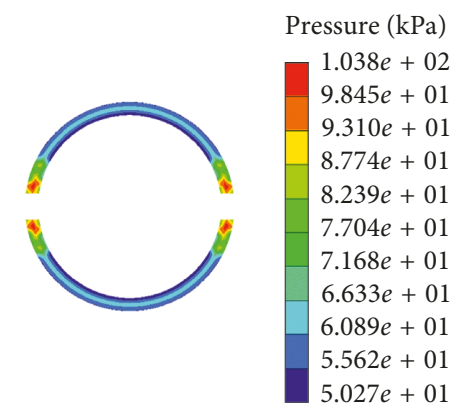

(a)

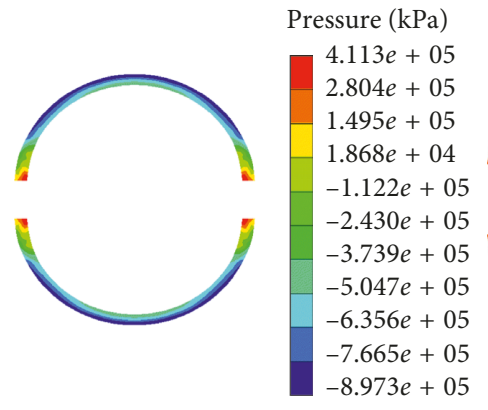

(d)

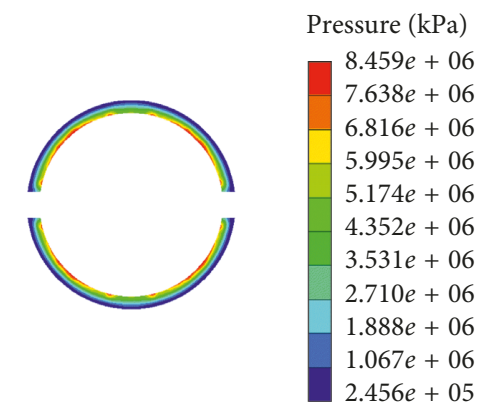

(b)

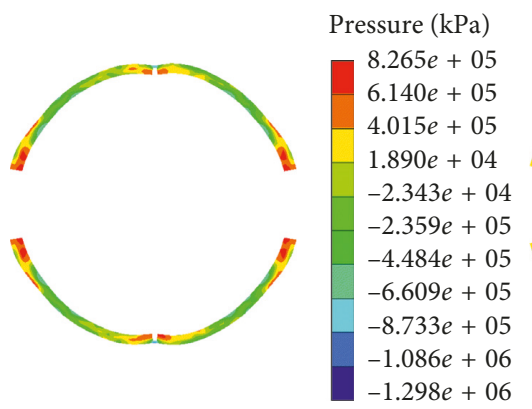

(e)

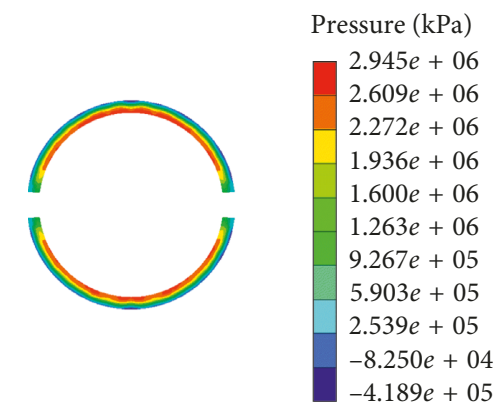

(c)

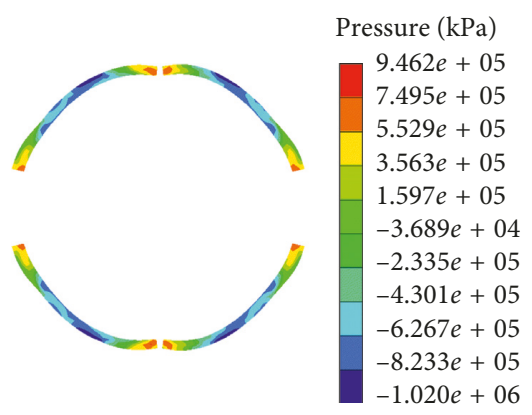

(f)

Figure 14: Dynamic pressure of split-tube charge.

inner wall of the cutting seam pipe. Wave incidence and reflection also occur at this time, and the rarefaction wave enters the explosion product, and the compressed blast wave is reflected to the cutting seam pipe. The process above is repeated throughout explosion process until the cutting seam pipe breaks, with initial breakage at the top of the cutting seam pipe.

\section{Conclusion}

The blast wave and detonation gas of cutting seam cartridge were tracked with a high-speed schlieren test system, allowing the separate study of these two actions. The explosion blast wave and detonation gas are highly symmetrical in expansion. The explosion blast wave first propagated along the cutting seam direction and then there is blast wave movement in other directions due to wave streaming and expansion. The state of the expansion blast wave changes from a dumb bell shape to an oval shape and propagates towards the air. The detonation gas initially expands from the cutting seam and then expands in an I-shaped form along the cutting seam direction. The detonation gas expands within a limited range along the vertical cutting seam 
direction. Initially, the detonation gas closely follows the blast wave front and then later the detonation gas and blast wave front slowly separate.

In the area close to the cutting seam cartridge, the pressure ratio between the cutting seam direction and vertical cutting seam direction decreases with the increase of the proportion distance. Additionally, the amplitude decreased with the increase of proportion distance. This suggests that the closer to the cutting seam cartridge, the larger the pressure difference of the air blast wave in these two directions. The blast wave change of the overall flow field of the cutting seam cartridge was numerically simulated, and the results were basically consistent with the high-speed schlieren test results in the distribution form. During expansion of the cutting seam pipe under the blast wave effect, the inner wall of the cutting seam pipe is mainly subject to tensile stress. When the tension force on the inner wall of the cutting seam pipe reaches the local yield limit, the cutting seam pipe wall will fail.

Overall, the experimental study on the blast wave of cutting seam cartridge suggests that the interaction between the cutting seam pipe and explosion blast wave can effectively control the release of explosion energy, enhance the effects of the blast wave and detonation gas on the medium in the cutting seam direction, and weaken the effects in the noncutting seam direction.

\section{Data Availability}

The data used to support the findings of this study are available from the corresponding author upon request.

\section{Conflicts of Interest}

The authors declare that they have no conflicts of interest.

\section{Acknowledgments}

This research was supported by the State Key Development Program for Basic Research of China (no. 2016YFC0600903) and Institutions of Higher College Discipline Innovation Plan "111 project" of China (no. B14006).

\section{References}

[1] Y. M. Zhang, Y. F. Yuan, and Q. Zhang, "The slit-charge breaking rock mechanism and application," Explosive $\mathrm{Ma}$ terials, vol. 30, no. 5, pp. 5-8, 2001.

[2] Y. Q. Yang, Q. K. Jin, R. S. Yang et al., "A new technology of directional fracture blasting in rock drift," Engineering Blasting, vol. 81, no. 1, pp. 8-10, 1995.

[3] V. K. Singh and D. P. Singh, "Controlled blasting in an openpit mine for improved slope stability," Geotechnical and Geological Engineering, vol. 13, no. 1, pp. 51-57, 1995.

[4] W. L. Fourney, J. W. Dally, and D. C. Holloway, "Controlled blasting with ligamented charge holders," International Journal of Rock Mechanics and Mining Sciences \& Geomechanics Abstracts, vol. 15, no. 3, pp. 121-129, 1978.

[5] W. L. Fourney, D. B. Barker, and D. C. Holloway, "Model studies of well stimulation using propellant charges,"
International Journal of Rock Mechanics and Mining Sciences \& Geomechanics Abstracts, vol. 20, no. 2, pp. 91-101, 1983.

[6] R. S. Yang, J. J. Zuo, Z. W. Yue et al., "Experimental study on opposite cracks propagation behavior under blast loading," Journal of China Coal Society, vol. 42, no. 5, pp. 1093-1098, 2017.

[7] R. S. Yang, Y. B. Wang, H. J. Xue et al., "SEM experiment of rock crack cross section morphology after explosion fracturing with slotted cartridge," Journal of China University of Mining and Technology, vol. 42, no. 3, pp. 337-341, 2013.

[8] R. S. Yang and Y. B. Wang, "Experimental study of dynamic fracture effect of blasting crack in slotted cartridge decoupling charge blasting," Chinese Journal of Rock Mechanics and Engineering, vol. 32, no. 7, pp. 1337-1343, 2013.

[9] R. S. Yang, Y. L. Che, D. K. Feng et al., "Tests for blasting vibration reduction technique with presplitting blasting of a slotted cartridge," Journal of Vibration and Shock, vol. 33, no. 12, pp. 7-14, 2014.

[10] Y. Wang, "Study of the dynamic fracture effect using slotted cartridge decoupling charge blasting," International Journal of Rock Mechanics and Mining Sciences, vol. 96, no. 5, pp. 34-46, 2017.

[11] K. Wang, Z. Q. Shi, Y. J. Shi et al., "Characteristics of electrical explosion of single wire in a vacuum and in the air," Acta Physica Sinica, vol. 66, no. 18, article 185203, 2017.

[12] K. Wang, Z. Shi, Y. Shi et al., "Characteristics of the electrical explosion of fine metallic wires in vacuum," AIP Advances, vol. 7, no. 9, article 095002, 2017.

[13] K. Wang, Z. Shi, and Y. Shi, "The formation and evolution of the core-corona structure in the electrical explosion of aluminum wire in vacuum: experimental and numerical investigations," Journal of Physics D: Applied Physics, vol. 50, no. 31, article 315201, 2017.

[14] L. C. Forde, W. G. Proud, S. M. Walley, P. D. Church, and I. G. Cullis, "Ballistic impact studies of a borosilicate glass," International Journal of Impact Engineering, vol. 37, no. 5, pp. 568-578, 2010.

[15] M. Kellenberger and G. Ciccarelli, "Simultaneous schlieren photography and soot foil in the study of detonation phenomena," Experiments in Fluids, vol. 58, no. 10, pp. 2-13, 2017.

[16] M. Kellenberger and G. Ciccarelli, "Propagation mechanisms of supersonic combustion waves," Proceedings of the Combustion Institute, vol. 35, no. 2, pp. 2109-2116, 2014.

[17] L. Li, J. Li, C. J. Teo, P. H. Chang, and B. C. Khoo, "Experimental study on incident wave speed and the mechanisms of deflagration-to-detonation transition in a bent geometry," Shock Waves, vol. 28, no. 2, pp. 205-216, 2018.

[18] L. Li, J.-M. Li, V. B. Nguyen, C. J. Teo, P.-H. Chang, and B. C. Khoo, "A study of detonation re-initiation through multiple reflections in a 90-degree bifurcation channel," Combustion and Flame, vol. 180, no. 3, pp. 207-216, 2017.

[19] J. Damazo and J. E. Shepherd, "Observations on the normal reflection of gaseous detonations," Shock Waves, vol. 27, no. 4, pp. 795-810, 2017.

[20] K. Mazaheri, Y. Mahmoudi, M. Sabzpooshani, and M. I. Radulescu, "Experimental and numerical investigation of propagation mechanism of gaseous detonations in channels with porous walls," Combustion and Flame, vol. 162, no. 6, pp. 2638-2659, 2015.

[21] Y. Mahmoudi, K. Mazaheri, and S. Parvar, "Hydrodynamic instabilities and transverse waves in propagation mechanism of gaseous detonations," Acta Astronautica, vol. 91, no. 10, pp. 263-282, 2013. 
[22] K. Mazaheri and S. A. Hashemi, "The effect of chain initiation reaction on the stability of gaseous detonations," Combustion Science and Technology, vol. 179, no. 8, pp. 1701-1736, 2007.

[23] S. I. Gerasimov, A. L. Mikhailov, and N. A. Trepalov, "Shock wave distribution in an explosion of an explosive material with plastic filler," Combustion, Explosion, and Shock Waves, vol. 53, no. 6, pp. 689-695, 2017.

[24] L. Larcher, M. Arrigoni, C. Bedon et al., "Design of blastloaded glazing windows and facades: a review of essential requirements towards standardization," Advances in Civil Engineering, vol. 2016, Article ID 2604232, 14 pages, 2016.

[25] X. Liu and L. Liu, "An immersed transitional interface finite element method for fluid interacting with rigid/deformable solid," Engineering Applications of Computational Fluid Mechanics, vol. 13, no. 1-3, pp. 337-358, 2019. 


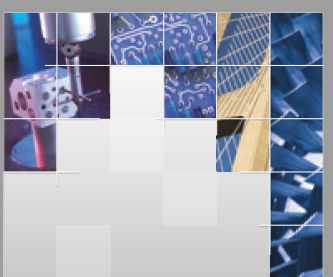

\section{Enfincering}
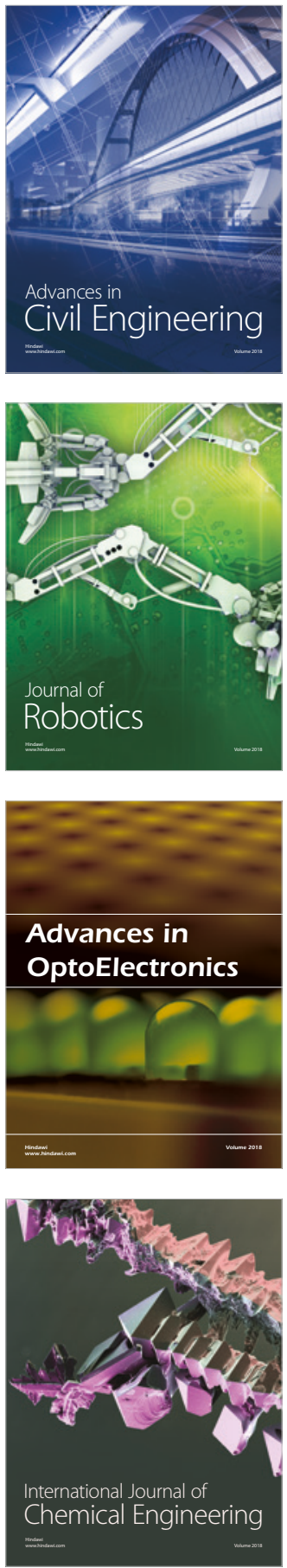

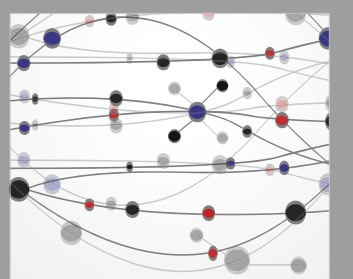

\section{Rotating \\ Machinery}

The Scientific World Journal

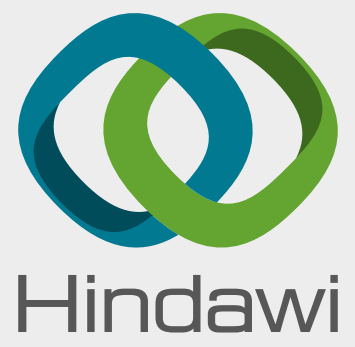

Submit your manuscripts at

www.hindawi.com
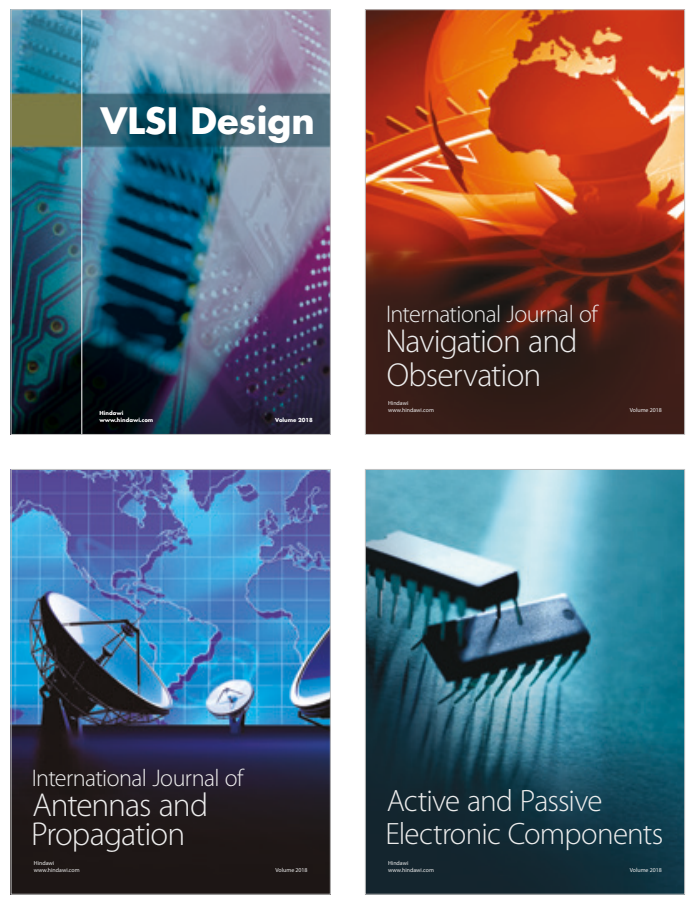
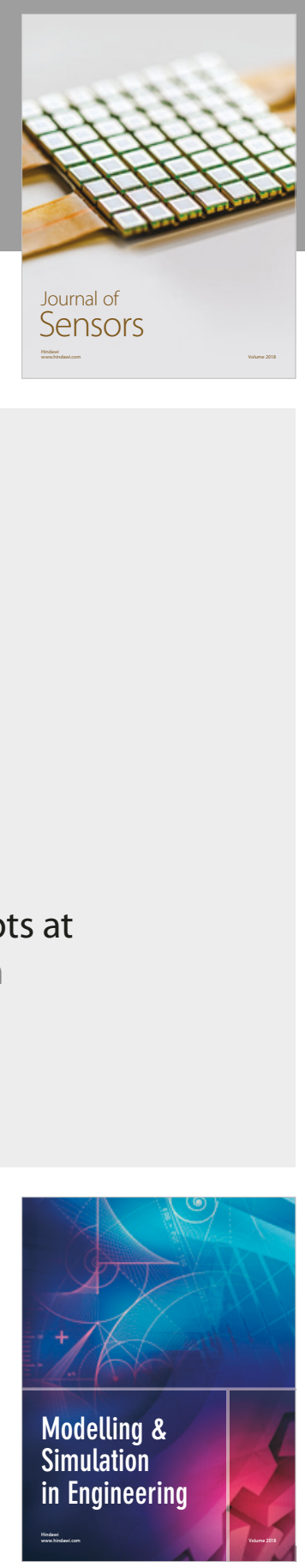

\section{Advances \\ Multimedia}
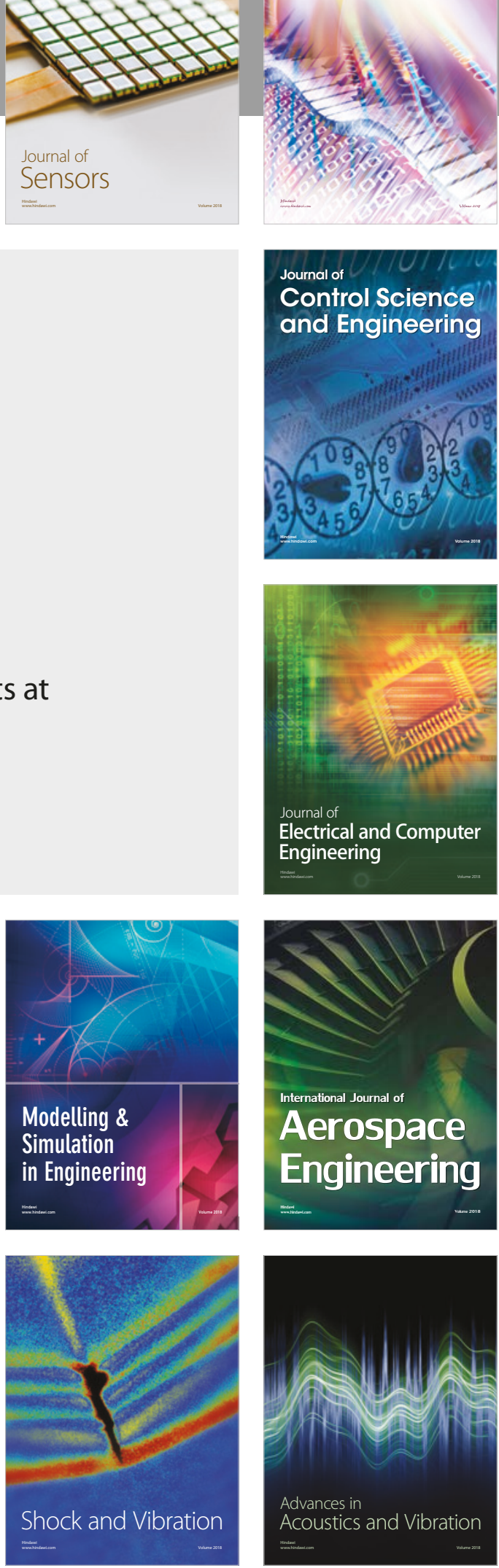